Case Reports
in Dermatology

\title{
Respiratory Distress Syndrome Associated with Erythrodermic Psoriasis
}

\author{
Lamya Noure ${ }^{a, b} \quad K^{2}$ irley Küçük ${ }^{c}$ Sylvain Raoul Simeni Njonnou ${ }^{b, d}$ \\ Véronique Del Marmol ${ }^{c}$ Jonathan M. White ${ }^{c}$ Benjamin Bondue ${ }^{a}$ \\ aDepartment of Pneumology, Hôpital Erasme, Université Libre de Bruxelles, Brussels, \\ Belgium; 'bepartment of Internal Medicine, Hôpital Erasme, Université Libre de Bruxelles, \\ Brussels, Belgium; 'Department of Dermatology, Hôpital Erasme, Université Libre de \\ Bruxelles, Brussels, Belgium; dDepartment of Internal Medicine and Specialties, Faculty of \\ Medicine and Pharmaceutical Sciences, University of Dschang, Dschang, Cameroon
}

\section{Keywords}

Acute respiratory distress syndrome · Erythrodermic psoriasis - Ciclosporin · Capillary leak syndrome $\cdot$ Psoriasis-associated aseptic pneumonitis

\begin{abstract}
Erythrodermic psoriasis is an uncommon and severe variant of psoriasis which may be associated with rare and severe complications such as acute respiratory distress syndrome. Early recognition of this life-threatening condition can allow prompt appropriate treatment. We report the case of a 69-year-old man with a long history of psoriasis who developed acute respiratory distress during a disease flare-up. There was no relevant past history (except for mild emphysema), known allergy, or recent treatment. Chest $X$-ray revealed new bilateral infiltrates, confirmed at chest computed tomography scan. Repeated cultures on aspirate of the bronchoalveolar lavage remained negative for viruses, bacteria, and parasites. Cardiac ultrasound was normal and high-dose corticosteroid therapy was initiated. Within a few days his clinical and radiological status improved significantly.

(C) 2021 The Author(s) Published by S. Karger AG, Basel
\end{abstract}




\section{Case Reports in Dermatology}

Case Rep Dermatol 2021;13:83-88

DOI: $10.1159 / 000511368$

\section{Introduction}

Psoriasis is a common chronic inflammatory skin disease affecting approximately $2 \%$ of the world population, varying according to region [1]; severe complications are rare. Vascular endothelial growth factor (VEGF) is responsible for angiogenesis within plaques of psoriasis [2] and is increased in erythrodermic psoriasis [3]. Erythrodermic psoriasis, the least common form of psoriasis, is characterized by erythema of $>75 \%$ of the body surface area with prolonged duration and hemodynamic, metabolic, and thermal disturbance [4]. Acute respiratory distress syndrome (ARDS) is an acute lung injury with bilateral diffuse pulmonary opacities, an alteration of gas exchange with a $\mathrm{PaO}_{2} / \mathrm{FiO}_{2}$ ratio $\leq 300 \mathrm{~mm} \mathrm{Hg}$, and absence of an argument for a rise in pressure in the left atrium (pulmonary artery occlusion pressure $\leq 18 \mathrm{~mm} \mathrm{Hg}$ ) [5]. ARDS can very rarely complicate generalized psoriasis (psoriasis-associated aseptic pneumonitis [PAAP]) and may be related to a pulmonary capillary leak syndrome (CLS) [6, 7]. CLS occurs when there is an increase in capillary permeability to proteins that are found in the interstitial space of the lungs. Cardiogenic pulmonary edema must be excluded and exudative serous cavity effusion and multiorgan involvement may be seen [8]. The precise role of a concomitant drug in the pathogenesis of PAAP is unclear, although reports of CLS associated with ciclosporin, acitretin, and other drugs have been published $[9,10]$. We present a case of ARDS in a patient with severe erythrodermic psoriasis despite phototherapy, for which ciclosporin therapy had recently been initiated.

\section{Case Presentation}

A 69-year-old man presented with a flare of chronic plaque psoriasis worsening over the last 18 months. There was no relevant past history (except for mild emphysema), known allergy, or recent treatment. Narrow-band UVB phototherapy was proposed. Treatment was discontinued after five sessions due to the worsening of lesions and the occurrence of a burning sensation, and he sought advice at the emergency department. Clinical examination showed erythema of $>90 \%$ of the body surface area with diffuse desquamation (Fig. 1) and mild edema of the extremities.

On admission, he had normal vital parameters and a blood pressure of $110 / 60 \mathrm{~mm} \mathrm{Hg}$. Biological exams at entrance were normal, except for a mildly increased CRP $(21 \mathrm{mg} / \mathrm{dL}$, nor$\mathrm{mal}<10 \mathrm{mg} / \mathrm{dL}$ ). Chest X-ray was normal at admission (Fig. 2a). A skin biopsy was performed, compatible with a diagnosis of psoriasis and essentially excluding alternative diagnoses such as Sézary syndrome (Fig. 3). The erythroderma was presumed to be secondary to phototherapy and was treated with local clobetasol propionate $0.05 \%$ and oral ciclosporin at $2.5 \mathrm{mg} /$ $\mathrm{kg} /$ day (150 mg/day) with initial improvement. On day 4 of admission, the patient developed ARDS with hypoxemia. He was apyrexial and had no cough. Clinical examination revealed bilateral crackles at both lung bases and worsening lower limb edema. There was no hepatojugular regurgitation or jugular vein distension. Blood gas analysis showed $\mathrm{pH} 7.47$, severe hypoxemia $\left(\mathrm{pO}_{2}\right.$ at $44 \mathrm{~mm} \mathrm{Hg}$ ), hypocapnia secondary to hyperventilation $\left(\mathrm{pCO}_{2}\right.$ at $27 \mathrm{~mm} \mathrm{Hg}$ ), and a lactate level at $2.3 \mathrm{mmol} / \mathrm{L}$ (normal $<2 \mathrm{mmol} / \mathrm{L}$ ). Chest X-ray revealed new bilateral lungs infiltrates (Fig. 2b). He was transferred to the ICU where noninvasive ventilation with continuous positive airway pressure was performed. Biological investigations revealed leu-

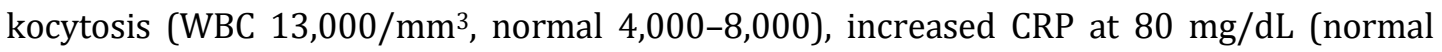
$<10 \mathrm{mg} / \mathrm{dL})$, normal renal function, elevated D-dimer $(2,988 \mathrm{ng} / \mathrm{dL}$, normal $<500 \mathrm{ng} / \mathrm{dL})$, and

\section{Karger'=}


elevated VEGF $(707 \mathrm{pg} / \mathrm{mL}$, normal $<500 \mathrm{pg} / \mathrm{mL}$ ). Bronchoalveolar lavage (BAL) and aspiration were performed. Cellular analysis showed an increased neutrophil count (59\%) contrasting with a normal lymphocyte count (2\%). The direct examination and culture of BAL fluid were negative. Pharynx cultures were negative for bacteria or viruses. Thoracic computed tomography (Fig. 4a) showed bilateral ground-glass infiltrates with alveolar condensation and excluded pulmonary embolism. Cardiac ultrasound was normal with no sign of cardiac dysfunction, cardiomegaly, overload, or elevation of filling pressure. A diagnosis of PAAP on the background of psoriasis was made and treatment with a high dose of systemic corticosteroids ( $2 \mathrm{mg} / \mathrm{kg}$ of methylprednisolone) was initiated $24 \mathrm{~h}$ after the symptoms had began. Ciclosporin was also stopped. Clinical improvement was observed with oxygen shutdown on day 7 after ICU admission. Favorable evolution was observed with progressive oxygen shutdown and progressive tapering of corticosteroids (at discharge from the hospital, the dose of methylprednisolone was $8 \mathrm{mg} /$ day). Two months later, the patient's lung lesions had returned to baseline (Fig. 4b) and his psoriasis was well controlled on topical treatments alone.

\section{Discussion}

We describe a case of severe ARDS in a patient with post-phototherapy psoriatic erythroderma initially treated with topical corticosteroid and oral ciclosporin. At the onset of ARDS, respiratory tract infection and cardiogenic pulmonary edema were rapidly excluded by BAL, cultures, and cardiac ultrasound. The role of ciclosporin in the pathogenesis of this case is not completely clear. Several cases of ARDS and CLS have been reported after intravenous injection of ciclosporin, but the causal agent there was probably the excipient (Cremaphor) rather than the ciclosporin (active ingredient) itself [10]. In that case, ARDS resolved after changing to oral ciclosporin. However, one case of ARDS related to oral ciclosporin was reported in 1988, where respiratory distress was linked to high blood levels of ciclosporin [11]. In our case, we feel that it is unlikely that ciclosporin played a causal role because of the low dose of ciclosporin administered and also the fact that the erythroderma and leg edema (probably an early sign of CLS) began before ciclosporin was commenced. Therefore, the most plausible diagnosis was PAAP.

PAAP is a rare complication of severe psoriasis. Given low overall numbers, there are no data on the epidemiology of ARDS in psoriasis, but there are a few isolated case reports [6]. Infectious pneumonia, congestive heart failure, pulmonary embolism, or drug-induced hypersensitivity (acitretin, cyclosporine, or anti-TNF blockers) should be excluded [10-12]. The pathophysiology of PAAP includes vascular changes responsible for systemic CLS. McGregor et al. [7] hypothesized a role of cytokines in the case of generalized pustular psoriasis (GPP), based on the relationship between the elevation of cytokine levels in psoriatic patients in particular (TNF- $\mu$ and IL-2) and the role of cytokines in the pathogenesis of CLS. Creamer et al. [3] found elevated plasma VEGF levels in patients with GPP. Recent studies found increased expression of VEGF and ICAM-1 in patients with erythrodermic psoriasis and GPP $[13,14]$. Our patient presented increased plasma VEGF titers, which favor hyperpermeability, although VEGF is not specific to psoriasis.

The treatment of PAAP is based on oxygen supply and high-dose corticosteroids, which reduces length of ICU stay and mortality [6]. In most of the cases, patients receive antibiotics before or in association with corticosteroid treatment, even with negative cultures, due to the

\section{Karger'=}




\section{Case Reports in Dermatology}

concomitant immunosuppression. In some cases, patients received ciclosporin and acitretin together, which were also effective with a less rapid response [6].

\section{Conclusion}

ARDS associated with psoriasis is a rare but potentially fatal complication whose pathophysiology is not well understood but probably related to CLS. Dermatologists should be aware of this complication, in order to closely monitor their patients with erythroderma, to allow rapid treatment with corticosteroids and to improve prognosis.

\section{Statement of Ethics}

Written informed consent was obtained from the patient for publication (including images). The study complied with the Declaration of Helsinki.

\section{Conflict of Interest Statement}

The authors have no conflicts of interest.

\section{Funding Sources}

Erasmus Hospital.

\section{Author Contributions}

L. Noure: first author of the manuscript. K. Küçük: co-author for the part on dermatology. V. Del Marmol, J.M. White: reviewed and corrected the dermatologic part. S.R. Simeni Njonnou: contributed with the section on internal medicine. B. Bondue: did a great diagnostic, corrected the manuscript, and supervised the whole work.

\section{References}

1 Parisi R, Symmons DP, Griffiths CE, Ashcroft DM; Identification and Management of Psoriasis and Associated ComorbidiTy (IMPACT) project team. Global epidemiology of psoriasis: a systematic review of incidence and prevalence. J Invest Dermatol. 2013 Feb;133(2):377-85.

2 Rendon A, Schäkel K. Psoriasis Pathogenesis and Treatment. Int J Mol Sci. 2019 Mar;20(6):1-28.

3 Creamer D, Allen M, Jaggar R, Stevens R, Bicknell R, Barker J. Mediation of systemic vascular hyperpermeability in severe psoriasis by circulating vascular endothelial growth factor. Arch Dermatol. 2002 Jun;138(6):791-6.

4 Jullien D, Villani A. 10-13 - Psoriasis. In: Saurat JH, Lipsker D, Thomas L, Borradori L, Lachapelle JM, editors. Dermatologie et Infections Sexuellement Transmissibles (Sixième Édition). Paris: Elsevier Masson; 2017. p. 533-44.

5 Chabot F, Papazian L, Guerin C, Roche N. Respiratory distress syndrome. Evidence based respiratory medicine: 2nd update workshop of the SPLF. Rev Mal Resp. 2004 Sep;21(4 Pt 1):860-2. French.

\section{Karger'=}




\section{Case Reports in Dermatology}

\begin{tabular}{l|l}
\hline DOI: $10.1159 / 000511368$ & $\begin{array}{l}\text { C } 2021 \text { The Author(s). Published by S. Karger AG, Basel } \\
\text { www.karger.com/cde }\end{array}$ \\
\hline
\end{tabular}

Noure et al.: Respiratory Distress Syndrome Associated with Erythrodermic Psoriasis

6 Kluger N, Bessis D, Guillot B, Girard C. Acute respiratory distress syndrome complicating generalized pustular psoriasis (psoriasis-associated aseptic pneumonitis). J Am Acad Dermatol. 2011 Jun;64(6):1154-8.

7 McGregor JM, Barker JN, MacDonald DM. Pulmonary capillary leak syndrome complicating generalized pustular psoriasis: possible role of cytokines. Br J Dermatol. 1991 Nov;125(5):472-4.

8 Siddall E, Khatri M, Radhakrishnan J. Capillary leak syndrome: etiologies, pathophysiology, and management. Kidney Int. 2017 Jul;92(1):37-46.

9 Vos LE, Vermeer MH, Pavel S. Acitretin induces capillary leak syndrome in a patient with pustular psoriasis. J Am Acad Dermatol. 2007 Feb;56(2):339-42.

10 Mackie FE, Umetsu D, Salvatierra O, Sarwal MM. Pulmonary capillary leak syndrome with intravenous cyclosporin A in pediatric renal transplantation. Pediatr Transplant. 2000 Feb;4(1):35-8.

11 Walsh RF, Springate JE, Spivack BS, Mosovich LL, Anthone R, Anthone S, et al. Cyclosporine and adult respiratory distress syndrome. Transplantation. 1988 Nov;46(5):776-7.

12 Atzeni F, Grillo E, Masala IF, Sarzi-Puttini P, Jones GT. Do Anti-TNF Blockers Increase the Risk of Lung Involvement in Patients with Ankylosing Spondylitis or Psoriatic Arthritis? A Systematic Review. Isr Med Assoc J. 2016 Mar-Apr;18(3-4):154-5.

13 Bressan AL, Pereira D, Medeiros PM, Carneiro S, Azulay-Abulafia L. How relevant are vascular endothelial growth factor and intercellular adhesion molecule in the systemic capillary leak syndrome of psoriasis? An Bras Dermatol. 2017 Nov-Dec;92(6):826-9.

14 Bressan AL, Picciani BL, Azulay-Abulafia L, Fausto-Silva AK, Almeida PN, Cunha KS, et al. Evaluation of ICAM1 expression and vascular changes in the skin of patients with plaque, pustular, and erythrodermic psoriasis. Int J Dermatol. 2018 Feb;57(2):209-16.

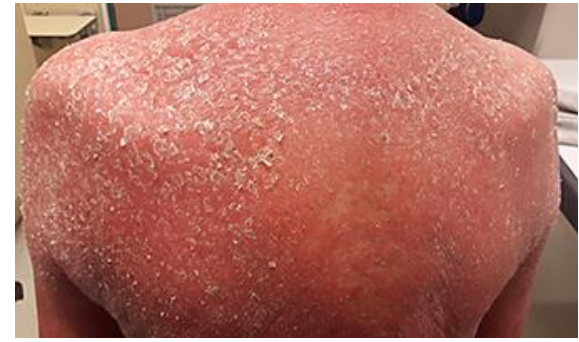

Fig. 1. Photograph illustrating the erythrodermic lesions with diffuse desquamation.

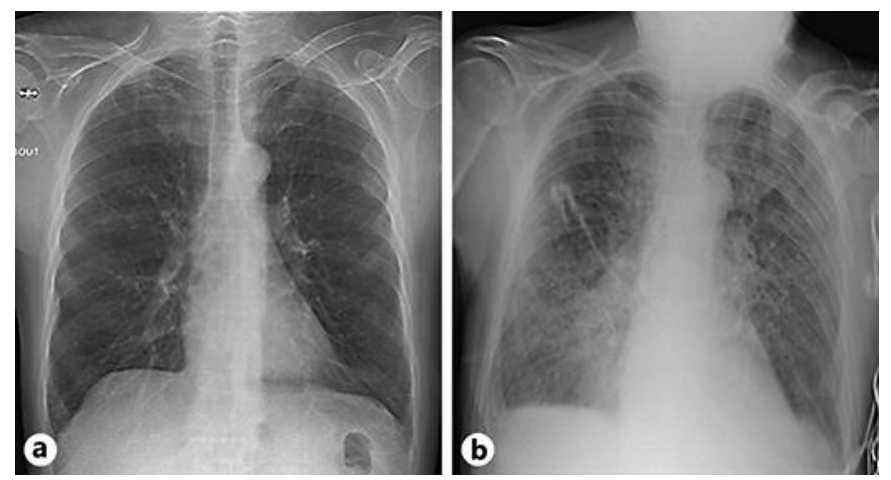

Fig. 2. Chest X-rays obtained at admission (a) and at day 4 (b) with new diffuse bilateral pulmonary infiltrates and bilateral mild pleural effusion without cardiomegaly. 


\section{Case Reports in Dermatology}

Noure et al.: Respiratory Distress Syndrome Associated with Erythrodermic Psoriasis

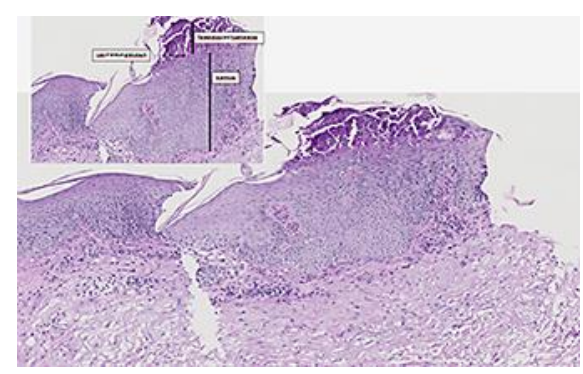

Fig. 3. Pathological examination of the skin biopsy (hematoxylin and eosin) showing the histological picture of psoriasis with granular layer loss, acanthosis, parakeratosis, and absence of spongiosis.

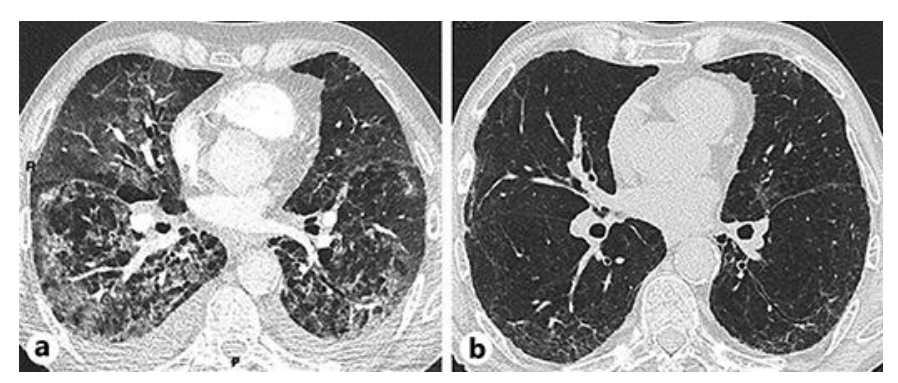

Fig. 4. Computed tomography scan of the chest showing severe bilateral interstitial infiltrates with groundglass opacities, condensations, and mild bilateral pleural effusion. After 2 months of corticosteroid therapy, pulmonary infiltrates and pleural effusions had disappeared (a), whereas emphysema changes were still observed (b). 